Aus der chirurgischen Abteilung des Stadtkrankenhauses Dresden-Johannstadt (Dirig. Arzt: Dr. Credé).

\title{
Symmetrische Lipombildungen.
}

\author{
Von Dr. Erich Zacharias.
}

(Mit 3 Abbildungen.)

Symmetrisch angeordnete Lipome sind nicht sehr häufig beschrieben worden. Eine Zusammenstellung der bekannten Fälle, die zur Erörterung der Frage der Entstehung dieser Geschwülste von Wichtigkeit ist, fehlt überhaupt noch.

Wenn das einzelne Lipom schon nicht zu den Alltäglichkeiten der chirurgischen Praxis gehört (Grosch), so trifft dies noch mehr für die symmetrischen Lipome zu, und ist die Literatur über das Wesen dieser Tumoren deshalb nicht so groß, wie vereinzelte Autoren glauben (Klinkowstein). In älteren Handbüchern der pathologischen Anatomie findet man nur kurze Erwähnungen dieser Geschwülste. Statistiken sind veröffentlicht von $\mathrm{L}$ anger, der 38 Fälle von multiplen symmetrischen Lipomen zusammenstellt. Sa ro u mian teilt ig Beobachtungen mit. Unter Iog Fällen, die Henningsen aus der Kieler Klinik bekannt gibt, findet sich ein einziges multiples symmetrisches Lipom, 2 kommen in der Statistik von Stoll vor, die sich auf I33 Lipome erstreckt. Jacobi sah im ganzen nur 5 Fälle. Schemensky, der die gesamte Literatur bis zum Jahre I9I2 einer genauen Durchsicht unterzog, nennt die Zahl der veröffentlichten Fälle ziemlich gering und gibt sie mit etwa 70 an. Dies dürfte wohl, soweit es sich übersehen ließ, die größte $\mathrm{Zu}$ sammenstellung sein. Die Mehrzahl der Beobachter verfügt über I-3 Fälle und hält sie bei der Seltenheit des Vorkommens der Veröffentlichung für wert. Eine Betrachtung dẹr einzelnen Statistiken ergibt jedenfalls die verhältnismäßige Seltenheit des Vorkommens der symmetrischen Lipome. 
Uber das Auftreten symmetrischer Lipome bei Tieren fand sich nur die Angabe von $\mathrm{Hutch}$ inson, wonach derartige Geschwülste bei Eseln nicht selten sind.

$\mathrm{Ob}$ einzelne Menschenrassen besonders zu Lipombildungen neigen, ist unbekannt. Nach Göbel sollen sie sich besonders häufig in England zeigen, was mit der dort häufigeren Arthritis zusammenhängen soll.

Die Mehrzahl aller Autoren spricht sich dahin aus, daß vor allem das weibliche Geschlecht mit Lipomen behaftet ist. Henningsen stellt für seine Io9 Fälle ein Überwiegen der Lipome bei Frauen fest. Grosch kommt ebenfalls zu diesem Ergebnis. Nach ihm kommen auf 254 mit Lipomen behafteten Männern 4II Frauen oder gleich auf Ioo Männer I62 Frauen. Der größere Fettansatz, der dem weiblichen Geschlecht eigen ist, wird dafür verantwortlich gemacht. Damit stimmt gut das Resultat der Untersuchungen von Sinnhuber überein, der eine Beteiligung der Frauen im Verhältnis zu den Männern wie I,6 zu I angibt. Schemensky bezweifelt diese Ansicht. Göbel behauptet, daß die Männer doppelt soviel als die Frauen an Lipomen, vor allem an symmetrischen leiden. Die größeren Schädlichkeiten, denen im allgemeinen das männliche Geschlecht durch Beruf und Lebensweise ausgesetzt sei, müßten als Gelegenheitsursache angesehen werden.

Jn jedem Lebensalter werden symmetrische Lipome beobachtet. Allerdings ist ihre Zahl in den ersten Lebensmonaten klein. 2o Proz. der von Plettner zusammengestellten Fettgeschwülste waren angeboren. Die überwiegende Mehrzahl der kongenitalen Lipome 'gehört nicht $z u$ den symmetrisch angeordneten (Hock). Mit zunehmendem Alter steigt auch die Häufigkeit; das mittlere Lebensalter, Anfang der 4oer Jahre, stellt den Höhepunkt des Auftretens dar (Schemensky). Bei Frauen hat besonders die Zeit der Pubertät und Menopause eine Bedeutung für die Entstehung von Fettgeschwülsten überhaupt und damit auch von symmetrischen.

In bezug auf die besondere Disposition einzelner Körperteile für das Entstehen von symmetrischen Lipomen sei erwähnt, daß am Schädel ihr Auftreten zu den größten Seltenheiten gehört. Clutton berichtet über einen Fall, in dem 
es sich um 2 symmetrische Tumoren am Hinterkopfe handelt neben vielen anderen am übrigen Körper. L ü cke erwähnt je ein Lipom in der Regio mastoidea bei einem 38 jährigen Mann, dasselbe hat Credé beobachtet. Schuster sah Fetttumoren hinter den Ohren mit Beteiligung vieler anderer Körperstellen. Vossi u s beschreibt einen Fall beiderseits am inneren Augenwinkel. Der Hals ist häufig der Sitz solcher Geschwülste. Etwa 30 diesbezügliche Beobachtungen sind in der Literatur niedergelegt. Wenn andere Körperteile Lipome in symmetrischer Anordnung enthalten, dann ist der Hals fast stets auch beteiligt. Strümpell beobachtete 2 walnußgroße Lipome am Nacken direkt an der Haargrenze in Verbindung mit gleichartigen Tumoren beider Oberarme und des Abdomens. Der Rücken ist selten symmetrisch befallen. Fischer sah, wie außer der Schultergegend der Rücken symmetrische Lipome trug. $\mathrm{Zu}$ beiden Seiten der Wirbelsäule saß je ein Lipom. Ferner sprechen Buchterkirch nud Bumke von multiplen symmetrischen Lipomen auf dem Rücken. Die Brustwirbelsäule im Bereiche des I. und 2. Wirbels war in dem Falle von Marimón betroffen mit Beteiligung des Halses, der Schulter- und Lendengegend. Häufiger als der Rücken wird die Hüft- und Gesäßgegend be: fallen, meist zusammen mit anderen Körperteilen. (F leis cher, Petrén, Mathieu und Spitzer). Ganz besonders of sind die Oberarme und Oberschenkel an der Bildung von symmetrischen Lipomen beteiligt. (Israel, Clutton, Köttnitz und Ha u m a n ). B o u cher d e Rou en sah multiple Lipome an den Händen. Die Oberarme sind öfter als die Unterarme befallen. Die oberen Extremitäten neigen mehr zu Lipombildungen als die unteren. Symmetrische Fußlipome sind nur von wenigen Autoren veröffentlicht. Köhler stellte einmal symmetrische Fibrolipome zwischen den Köpfchen des ersten und zweiten Metatarsalknochens fest, J a cksch erwähnt symmetrische Fettgeschwülste auf dem Fußrücken, Sprengel zwischen den Sehnenscheiden des Fußes. Pagenstecher verfügt über 2 Fälle, der eine davon betraf ein Kind mit angeborenen symmetrischen Lipomen am inneren Fußrande. Von weiteren Stellen der unteren Extremitäten kommen vor allem der Malleolus externus und die Kniekehle in Betracht. Wir sehen also, dal' alle Körperteile 
befallen sein können, daß sie sich aber in der Disposition voneinander unterscheiden. Im allgemeinen sollen Stellen, die Insulten ausgesetzt sind (Köttnitz), sowie drüsenarme Bezirke (Grosch) und Regionen, die durch starkes Fettlager sich auszeichnen, bevorzugt werden.

Sehr schwanken die symmetrischen Lipome in der $\mathrm{Zahl}$ ihres Vorkommens bei derselben Person. Meist ist ihre Zahl nicht groß, doch treten sie bisweilen auch sehr zahlreich auf. 76 nahezu symmetrische Lipome konnte Alsberg in einem Falle zählen, Curling 43 Stück, v on Wahl 25, außerdem einmal Io6, Spitzer 28. Die allerhöchsten Zahlen, die Hunderte, sogar einige Tausende von Tumoren umfassen, stammen nicht von streng symmetrisch angeordneten Lipomen, denn die Symmetrie wird dann durch' das regellose multiple Auftreten, das sich über den ganzen Körper erstreckt, aufgehoben. So beobachtete von L utza u 2436 Tumoren an einer Person.

Wie steht es nun mit dem Wachstum der symmetrischen Lipome? Meist ist es langsam und stetig, doch treten auch bis jahrelange Pausen im Wachstum ein, ohne daß wir die Gründe dafür kennen. Andere Lipome zeigen gleich von Beginn an ein beschleunigtes Wachstum (Klinkowstein). Sogar zu Fehldiagnosen kann das ungewöhnlich schnelle Wachstum ver. leiten und leicht zur Annahme einer bösartigen Geschwulst führen (Hodges). In seltenen Fällen geht die Größe spontan zurück. Eine bestimmte Ursache muß selbstverständlich stets dafür vorhanden sein. So kann die fortschreitende Carcinomkachexie verantwortlich gemacht werden in dem Falle von Petrén, der von einer Größenabnahme von Lipomen bei einer an Carcinoma pylori gestorbenen Frau berichtet. Leider finden sich in der Literatur äußerst wenig genaue Angaben über ein plötzliches Wachstum von symmetrischen Lipomen, ebenso gibt sie über Fälle, in denen symmetrische Lipome eine beachtenswerte Größe erreicht hätten, kaum Aufschluß. Bei Frauen hat man ein schnelleres Wachstum von Lipomen nach Sistieren der Menses, in der Menopause festgestellt.

In jedem Falle einer Geschwulstbildung ist die Frage eventueller Heredität wissenschaftlich interessant. Eine einzige Beobachtung direkter Vererbung von symmetrischen Lipomen 
Meine eigenen Beobachtungen von symmetrischen Lipomen beziehen sich auf folgende zwei Fälle.

Alice Schn., 9 jährig, bisher gesund, stammt von gesunden Eltern. Geschwulstbildungen sind bei den Eltern sowie Großeltern nicht zur Beobachtung gekommen. Bereits im Alter von einem Jahre wurde von der Mutter bei ihr eine Anschwellung der linken Hüfte

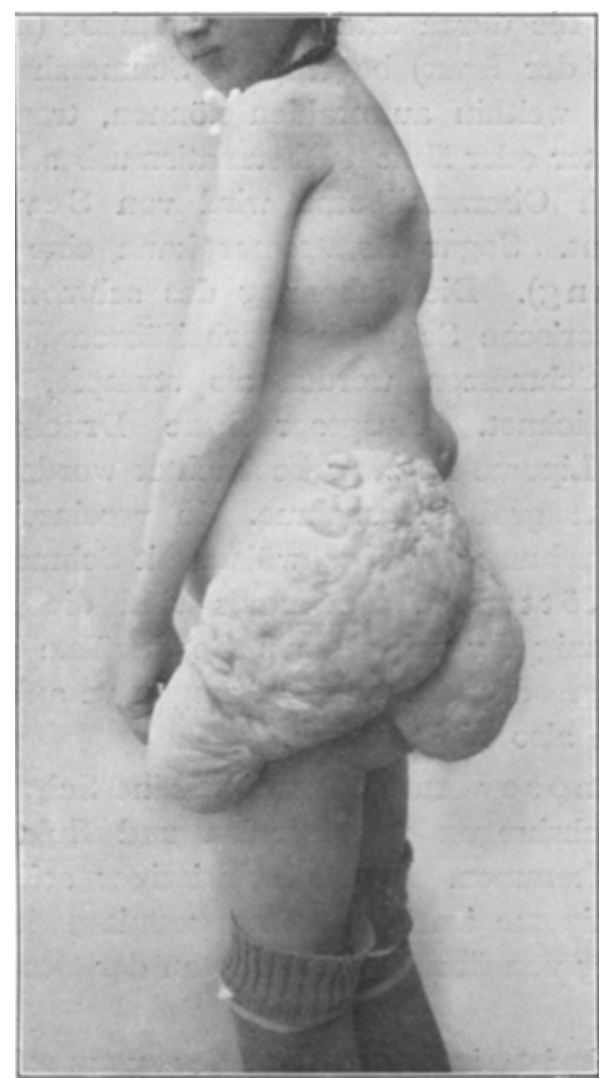

Fig. I.

bemerkt und eine Verdickung an der gleichen Stelle der rechten Seite. Schon damals stellte der Arzt Fettgeschwülste fest. Salbenverbände hatten keinen Erfolg. Vielmehr wuchsen die Lipome stetig, wenn auch langsam. Deshalb wurden die gesamten Geschwulstmassen operativ entfernt, als das Kind $21 / 2 \mathrm{Jahr}$ alt war. Die entfernten Lipome sollen damals nach Angabe der Mutter ein Gewicht von etwa 
ist, soweit es sich in der vorliegenden Literatur ersehen lieB, niedergelegt (Fall Ochsener).

Die Symptome, die die symmetrischen Lipome auslosen, sind abhängig von dem Sitz, der Größe und dem Gewicht derselben. Meist verursachen sie keinerlei Beschwerden und werden bei Untersuchungen zufällig entdeckt. Fast die einzige Folgeerscheinung ist die durch örtliche Verhältnisse (Druck auf Nerven, Spannung der Haut) begründete Schmerzhaftigkeit. Diese Schmerzen, die weithin ausstrahlen können, tragen oft neuralgischen Charakter oder ähneln Rheumatismusbeschwerden. Armneuralgie durch Oberarmlipome wird von Severanu sowie Is rael erwähnt. Sogar Armtremor kann eine weitere Folge sein (Madelung). Die Schonung des schmerzenden Gliedes kann eine motorische Schwäche herbeiführen (Is rael, Sangalli). Die Schmerzen werden als stumpfe, reißende oder stechende bezeichnet. Ausgesprochene Druckempfindlichkeit symmetrischer Lipome ist zwar beobachtet worden (Alsberg), bildet aber eine große Ausnahme. In vereinzelten Fällen ist bereits die Entstehung der Geschwülste mit Schmerzen verknüpft (Kleissel, Köttnitz). Jedenfalls sind die symmetrischen Lipome mit wenig Ausnahmen als Geschwülste anzusehen, die keine Schmerzen oder Gebrauchsstörungen veranlassen, das Allgemeinbefinden also nicht stören.

Die Diagnose verursacht meist keine Schwierigkeit, doch können Verwechslungen mit Hernien und fluktuierenden Geschwülsten vorkommen. Etwas schwieriger soll sich die Erkennung der Lipome im Kindesalter gestalten durch die reichliche Beteiligung von fibrösem Gewebe an der Geschwulstbildung (B u tlin).

Sehr günstig ist die Prognos e der symmetrischen Lipome, namentlich auch weil bösartige Entartungen nicht vorkommen.

Die Therapie richtet sich nach den Beschwerden und kosmetischen Gründen, sie kann nur eine operative sein. Entfettungskuren, die in vielen Fällen angewendet wurden, versprechen keinen Radikalerfolg (Hirsch). F le is cher, S te ys kel, Türk, Rothmann und Morsaline wollen von Anwendung von Thyreoideapräparaten Günstiges gesehen haben. 
2--3 Pfund auf jeder Seite besessen haben. Aber bereits 4 Jahre später hatten sich an der gleichen Stelle vor allem rechts, neue Tumoren gebildet, die bald größer waren als die ersten. Diese Geschwülste hinderten das Kind in hohem Grade beim Sitzen in der Schule, sie waren außerdem sehr störend beim Gehen durch Größe und Gewicht, das von der Mutter auf 5 Pfund geschätzt wird. Im Jahre 1913 wurde erneut eine Operation vorgenommen, die sich hauptsächlich auf die rechte Hüftgegend beschränkte. Ganz kurze Zeit darnach zeigten sich die Anfänge erneuten Wachstums. Die Größenzunahme erfolgte jetzt auf der linken Seite derart schnell, daß die Kranke am I7. V. I9I4, also 6 Monate nach der 2 Operation, im hiesigen Krankenhause wiederum zur operativen Entfernung der Lipome aufgenommen wurde.

B e f u n d: $142 \mathrm{~cm}$ großes Mädchen in mäßig gutem Ernährungszustande, keine Ödeme. Gewicht: $44 \mathrm{~kg}$. Urin: kein Eiweiß, kein Zucker. Haut sowie Schleimhäute blaß. Thyreoidea nicht vergrößert. Thorax: Herz und Lungen ohne Besonderheiten. Bauch: nicht druckempfindlich. Reflexe normal. An der rechten Hüfte verläuft in querer Richtung eine nicht druckempfindliche Narbe, die ihren Ausgang dicht unterhalb der Spina iliaca anterior superior rechts nimmt und sich über die Hüfte und den Rücken hinweg bis zu den großen Tumormassen der linken Hüfte erstreckt. Die direkte Umgebung dieser Narbe ist stark eingezogen, teils in der Narbe selbst als auch besonders dicht daneben zu beiden Seiten sind mehrere, sich flächenhaft ausbreitende Lipombildungen zu bemerken. Oberhalb der rechten Crista iliaca, neben der Lendenwirbelsäule gelegen, fühlt man einen über faustgroßen Tumor, der nicht druckempfindlich ist. Dieser Tumor ist gegen seine Umgebung nur schwer abzugrenzen, fühlt sich weich an, die Haut über ihm ist nicht abhebbar. Auf der diese Geschwulst deckenden Haut sitzen mehrere kleine, gut gestielte. An der gleichen Stelle links lateral von der Wirbelsäule ist eine etwa hühnereigroße Geschwulst $z \mathbf{u}$ tasten mit denselben Eigenschaften wie die rechtsseitige. Die ganze linke Hüftgegend ist mit Tumormassen besetzt. Diese bilden vornehmlich zwei große überhängende Lappen, denen ein dritter, kleinerer $19 \mathrm{~cm}$ großer und $14 \mathrm{~cm}$ breiter Lappen angegliedert ist. Die großen Lappen zeigen schön den für Lipome charakteristischen lappigen Bau. Besonders der dritte kleinere zerfällt völlig in kleine Läppchen. Diese drei Geschwülste sind durch Längsfurchen voneinander getrennt, sie nehmen gesondert voneinander ihren Ausgang. Die Haut über dem obersten Lappen ist wegen der Schwere der Geschwulst äußerst gespannt, die Venenzeichnung daselbst deutlich sichtbar. Tumoren selbst von weicher Konsistenz. Am größten ist der oberste Tumor. Er erstreckt sich um die ganze linke Hüfte herum bis zur Mitte des linken Oberschenkels. Die beigefügten Abbildungen, die ich Herrn Dr. P lenge 
verdanke, zeigen gut die enorme Größe. In der Umgebung dieses großen Tumors finden sich multiple, kleine gut gestielte. Der mittlere der drei großen Lappen nimmt die gesamte linke Gesäßgegend ein und ist über Manneskopf groß. Der dritte kleinere hängt direkt zwischen den beiden Glutäen vor dem Anus herab. Ein Teil davon

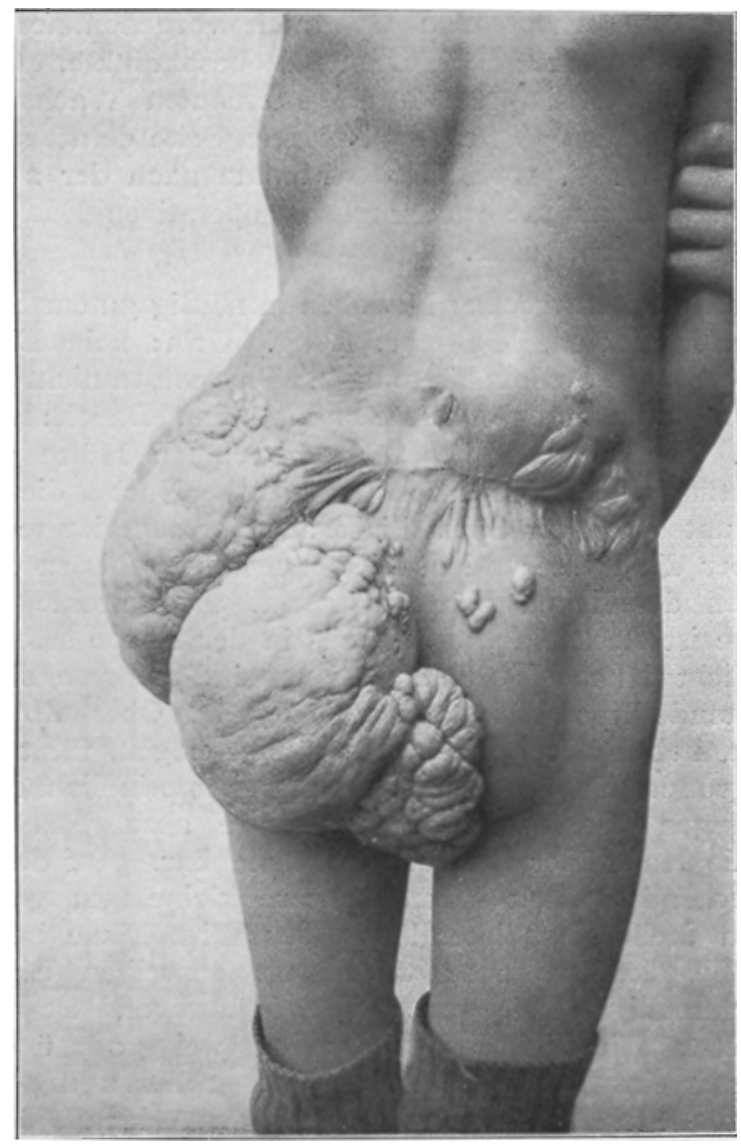

Fig. 2.

ist sogar auf der Vorderansicht zwischen beiden Beinen am Damm zu sehen. Bauchdecken der linken seitlichen Bauchgegend stark gespannt, ganzer Leib aufgetrieben. Im Bereiche der 7. bis 9. Rippe linksseitig lateral der Mammillarlinie fühlt man eine über faustgroße Geschwulst, die von ihrer Umgebung nur schwer abgrenzbar ist. Die Haut darüber leicht abhebbar, die Geschwulst selbst von weicher 
Konsistenz. Dicht darüber zieht unterhalb der linken Achselhöhle, am Rücken über das Schulterblatt hinweg bis zur Wirbelsäule im Bereich der mittleren Brustwirbel, eine große, diffuse Anschwellung, die sich bei näherer Untersuchung als ein weiterer Tumor mit den schon öfter gekennzeichneten Merkmalen herausstellt. Für diese beiden letzterwähnten Tumoren fehlen die symmetrischen Geschwulst. bildungen an den entsprechenden Stellen der rechten Seite. Beim

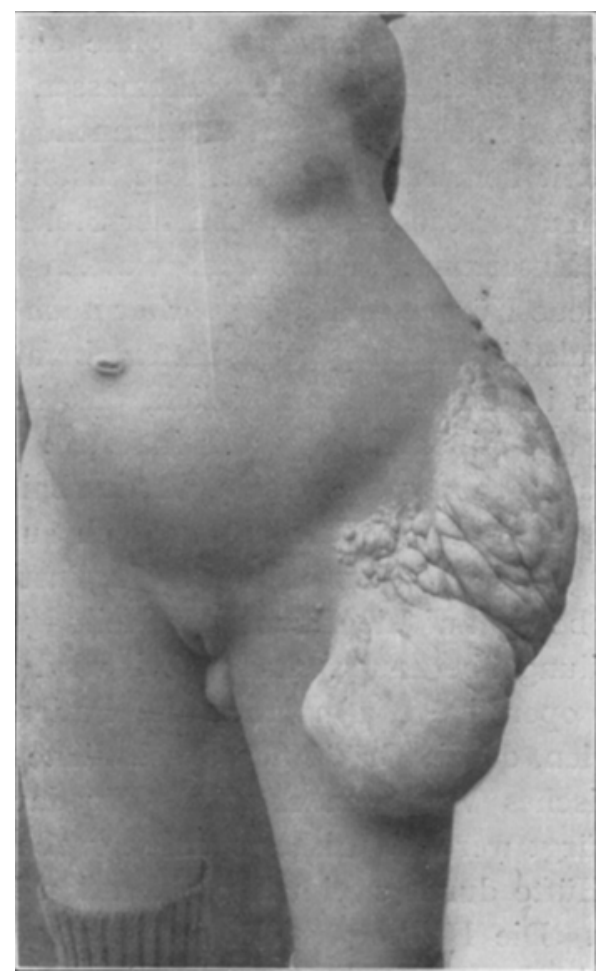

Fig. 3 .

Gehen hinkt die Patientin leicht mit dem linken Bein. Die linke Schulter steht deutlich tiefer als die rechte. Es macht den Eindruck, als ob die Schwere der Geschwülste die ganze linke Seite herabzöge. Über irgendwelche Schmerzen klagt Patientin nicht, Etwaige Entwicklungsstörungen sind nicht festzustellen.

Die Diagnose wurde auf symmetrische Lipome gestellt. Die Anlage dieser Lipome war streng symmetrisch. Denn an beiden Hüften hatten früher Lipombildungen bestanden. Nur hatte die radi: 
kale Entfernung der Tumoren der rechten Hüfte günstigeren Dauererfolg als linksseitig. Die beiden Lipome neben der Lendenwirbelsäule entsprechen sich völlig in ihrem Sitz. Diese auf Grund der Beobachtung und Untersuchung gewonnene Diagnose wurde durch die histologische Untersuchung mehrerer Tumorstücke sichergestellt (Prof. Dr. G e i p e l).

Wenn auch die Prognose der Lipome im allgemeinen sehr gut ist, so können wir sie für unseren Fall nicht für so günstig: ansehen. Zweimalige Operationen sind ohne dauernden Erfolg geblieben, im Gegenteil die Geschwulstmassen sind jetzt beträchtlicher als zuvor. Dazu kommt das schnelle Wachstum, das sich in letzter Zeit bemerkbar macht. Die histologischen Untersuchungen von Tumorstücken verschiedener Stellen ergaben aber keine maligne Entartung. Außerdem wurden in den letzten Monaten ganz neue Körperstellen, die vorher noch gar keinen Ansatz zu Geschwulstbildungen gezeigt hatten, befallen (linke Rippengegend). Es ist daher wahrscheinlich, daB in Zukunft noch andere Bezirke der Lipombildung anheimfallen. Diese Lipome, mögen sie an sich noch so gutartig sein, werden bei ihrer schnellen Größenzunahime sowie ihrer Verbreitung doch zu schweren Störungen des Allgemeinbefindens führen. Ungünstig ist die Prognose auch in bezug auf die Dauer der Heilung und die Möglichkeit der Heilung überhaupt. Lange Zeit wird es in Anspruch nehmen durch operative Maßnahmen die Patientin von ihrem Leiden zu befreien, da die Größe der Geschwülste nur ein schrittweises, chirurgisches Vorgehen erlaubt. Für dauernden Erfolg kann nicht gebürgt werden. Bisher wurden große Tumormassen an der linken Hüfte durch zweimalige Operation bei unserer $\mathrm{Pa}$ tientin entfernt. Die Heilung verlief jedesmal ganz glatt. Der Fall ist also sehr bemerkenswert durch die enorme Größe der Geschwülste, ihr symmetrisches Auftreten und ihre kongenitale Entstehung.

Der zweite Fall von symmetrischen Lipomen betrifft eine 7ojährige Frau, Dorothea K. Sie stammt aus gesunder Familie. Die Mutter starb über Ioo Jahre alt an Altersschwäche. Als Kind hatte die Patientin Keuchhusten, Masern und Röteln, später ist sie nicht ernstlich krank gewesen. Patientin suchte jetzt das Krankenhaus auf wegen Unterleibsbeschwerden mit ileusartigen Erscheinungen.

B ef und: Große, für ihr Alter sehr rüstige Frau in gutem Ernährungszustande, keine Ödeme. Thyreoidea nicht vergrößert. Re- 
flexe normal. Urin frei von Zucker und Eiweiß. Thorax: Herz und Lungen ohne Besonderheiten. Bauch: diffus mäßig stark druckempfindlich. Genitalbefund ohne Belang. Bei der Untersuchung wurden an beiden Vorderarmen mehrere kleine Tumoren entdeckt. Etwa in der Mitte des linken Vorderarmes fühlt man auf der Streckseite eine taubeneigroße Geschwulst. Sie ist von ihrer Umgebung scharf abgegrenzt, die darüberliegende Haut ist gut abhebbar. Ganze Geschwulst auf ihrer Unterlage leicht verschieblich, von weicher Konsistenz. Keinerlei Druckempfindlichkeit. Weitere Tumoren sind am linken Vorderarme nicht aufzufinden. Völlig symmetrisch damit ist an der gleichen Stelle des rechten Vorderarmes ein Tumor vorhanden, der an Größe und sonstigen Merkmalen völlig mit dem linksseitigen übereinstimmt. Außerdem ist am rechten Unterarm auf der Beugeseite handbreit unterhalb des Ellenbogengelenkes eine enteneigroße Geschwulst zu fühlen von derselben Beschaffenheit. Dicht darüber läßt sich noch ein haselnußgroßer Tumor, neben diesem wiederum ein kleinhühnereigroßer feststellen. Eine genaue Körperuntersuchung ergab keine weiteren Geschwülste gleichen Charakters. Auf Befragen gibt die Patientin an, daß sie bereits vor 25 Jahren an diesen Stellen Anschwellungen bemerkt habe. Damals seien sie kaum erbsengroß gewesen. Da sie niemals über irgendwelche Beschwerden zu klagen hatte, schenkte sie den Geschwülsten keine Beachtung. Diese wuchsen innerhalb dieser Zeit allmählich bis zu der beschriebenen Größe. Die Schwangerschaften, das Wochenbett und die Menopause führten kein schnelleres Wachstum herbei.

Die Diagnose symmetrischer Vorderarmlipome war also einwandfrei. Dic Prognose ist absolut günstig. $\mathrm{Zu}$ einer operativen Entfernung der Lipome wic überhaupt zu einer Behandlung konnte sich Patientin infolge der selbst beobachteten Harmlosigkeit der Geschwülste nicht entschließen.

Ähnliche Fälle sind mehrfach bekannt und in der Literatur niedergelegt. Die Seltenheit unseres Falles beruht auf der ausgesprochenen Heredität, die sich gut verfolgen läßt. Die Patientin gibt an, daß ihre Mutter die gleichen Geschwülste an beiden Vorderarmen jahrzehntelang besessen habe. Leider ist man in dieser Beziehung nur auf die Angaben der Patientin angewiesen. Nun bot sich die günstige Gelegenheit, die Tochter, die im 38. Lebensjahre steht, auf Lipome zu untersuchen. Wir konnten die äußerst interessante Beobachtung machen, daß auch sie auf beiden Vorderarmen symmetrisch angeordnete Lipome zeigte. Am linken Unterarm saßen zwei auf der Beuge- und zwei auf der Streckseite, am rechten Vorderarm drei auf der Beugeund zwei auf der Streckseite. Sämtliche Tumoren waren unge- 
fähr erbsengroß. Somit ist unser Fall einzigartig. Bisher ist erst eine Beobachtung veröffentlicht ( $O$ chsener), wo in absteigender Linie bei drei weiblichen Mitgliedern einer Familie streng symmetrische Lipome festgestellt wurden.

Über die Ätiologie der symmetrischen Lipome wissen wir noch fast nichts, nur Theorien gibt es. Hutchins on $z$. B. erblickt in den Halslipomen paläogenetische Entwicklungsanomalien auf dem Grunde der tierischen Mähne. Andere Autoren sprechen sich für einen Zusammenhang zwischen allgemeiner Fettleibigkeit und Lipombildungen aus. (Sinitzyn, Eckstein). Die Zahl der gegenteiligen Beobachtungen ist jedoch sehr groß. Strauß erwähnt ausdrücklich für seinen Fall von diffusen symmetrischen Lipomen bei gleichzeitiger florider Phthise die hochgradige Unterernährung. Schemensky stellte die multiple Lipomatose häufig bei sonst mageren, selbst körperlich heruntergekommenen skrofulösen Menschen fest. Für die Entstehung ist weiterhin der Einfluß eines erlittenen Traumas erwogen worden. Wohlgemerkt handelt es sich in diesen Fällen meist um solitäre Lipome. Wenn auch schon hierbei viele sich dagegen ausgesprochen haben und das 'Irauma nur als Gelegenheitsursache gelten lassen, so verliert für die Bildung von symmetrischen Lipomen das Trauma ganz an Bedeutung. Daß ein Fall mehrere Stockwerke herab die Bildung von symmetrischen Lipomen am Nacken, Oberarm und Leib zur Folge haben sollte (Fall Strümpell), ist sehr unwahrscheinlich. Strüm. pell spricht sich gleichfalls in diesem Sinne aus. Marimón beobachtete symmetrische Lipome am Rücken, Ilals und Lendengegend ein Jahr nach einer Fraktur der Brustwirbelsäule. Als direkte Ursache erblickt er jedoch nicht den Unfall selbst, sondern die durch das Trauma bewirkten trophoneurotischen Störungen. Im Anschluß hieran mag auch die Einwirkung von kontinuierlichem Druck gestreift werden. Kölliker erblickt in Druck und Reibung eine Gelegenheitsursache für die Entstehung von Lipomen. Es ist sogar der Ausdruck Drucklipom geprägt worden und das Lipom als eine Gewerbekrankheit für Lastträger, Müller. Fleischer usw. bezeichnet worden. Meist handelt es sich um solitäre Lipome, doch sind auch doppelseitige, namentlich auf den Schultern, beobachtet worden. Nach D or a n soll der Druck des 
Korsettes bei Frauen die Entstehung von Lipomen in der Lendengegend begünstigen. Schon bei der Besprechung der Therapie war erwähnt worden, daß3 mitunter bei dem Auftreten von symmetrischen Lipomen pathologische Verhältnisse der Schilddrüse vorliegen. Diesbezügliche Sektionsbefunde sind nur in geringer Zahl veröffentlicht. Curling konnte in zwei Fällen von symmetrischen Halslipomen bei der Sektion keinerlei Schilddrüsenanlage finden. Einen ähnlichen Befund teilt Madel ung mit. In dem Falle von Steyskel war die Schilddrüse ungemein dünn, außerdem bestand in der Hypophyse eine Cyste. Eine ausgesprochene Atrophie beider Schilddrüsenlappen war in dem von Fränkel publizierten Falle vorhanden, der ganze linke Schilddrüsenlappen fehlte in dem Falle von $\mathrm{Kla}$ a $B \mathrm{ner}$. F le is cher berichtet, daß er einmal bei symmetrischen Lipomen die Thyreoidea nicht palpieren konnte. Dagegen war ein cystischer Tumor zu fühlen, der vielleicht mit der Schilddrüse in Zusammenhang stand. Einige andere Autoren, z. B. Türk, Rothmann, Morsaline, Madelung, Klaußner, Köttnitz und $\mathrm{P}$ a y $\mathrm{r}$ sprechen ebenfalls von symmetrischen Lipombildungen bei fehlender oder schlecht entwickelter Schilddrüse. In allen diesen Fällen würde eine Dysfunktion im Sinne einer Hypofunktion vorliegen. Neuwelt beobachtete gleichzeitig Basedow und symmetrische Lipomatosis, Köttnitz Lipome und Struma an einer Person, ebenso Wolfsohn symmetrische Lipomatose und Struma. Letzterer nimmt einen Zusammenhang zwischen symmetrischen Lipomen und Hyperthyreoidismus an. Von den Vertretern einer ätiologischen Beziehung zur Schilddrüse können auch die Erfolge der Therapie mit Schilddrüsenpräparaten als Stuitze ihrer Anschaunng angeführt werden. Um berechtigt zu sein, in der durch eine Dysfunktion der Schilddrüse bewirkten Störung der inneren Sekretion eine Ursache für die Entstehung von symmetrischen Lipomen zu erblicken, dafür fehlen doch die exakten Beweise. Ähnlich verhält es sich mit dem Einfluß der Menopause. Auch da dürfte eine veränderte Wechselbeziehung des Eierstockes als Drüse mit innerer Sekretion ätiologisch heranzuziehen sein. In gleichem Sinne kann vielleicht die Entstehung der multiplen symmetrischen Lipome in dem Falle von $\mathrm{Schu}$ ster gedeutet werden, der die Entwicklung der Fettgeschwülste 
bei einem 47jährigen Manne im Anschluß an die Kastration sah. Der Funktionsausfall der Keimdrüsen bildet die Grundlage. Vorläufig stehen auch hier uns strenge Beweismittel nicht zu Gebote, so daß wir über den Begriff der Theorie nicht hinauskommen. Eine große Anzahl von Forschern berichtet über das gleichzeitige Auftreten von rheumatischen Affektionen aller Art und symmetrischen Lipomen. Besonders französische Autoren haben sich diesen Studien gewidmet und eine große Zahl derartiger Beobachtungen bekannt gegeben. Neuere Autoren erkennen rheumatische Leiden höchstens als Gelegenheitsursache an oder verwerfen jede ätiologische Beziehung. Auch der Alkohol ist von einigen Seiten als ursächliches Moment angeschuldigt worden, aber wohl ohne Grund. Wenn üppige Lebensweise als Ursache mitunter bezeichnet wurde, so ist diese Fassung so allgemein, $\mathrm{da} B$ es sich erübrigt, darauf einzugehen. Der Theorie von verirrten embryonalen Geschwulstkeimen sind ebenso wie sie Anhänger gefunden hat, auch Gegner entstanden. Vor allem führt Grosch gegen sie die gesetzmäßige Lokalisation der Lipome an. Borst weist bei multiplen Lipomen auf die Möglichkeit von Keimversprengungen hin. Am meisten haben die Anschauungen von Grosch Billigung gefunden, nach ihm sind die Disposition zur Lipombildung und ihre Lokalisation abhängig von dem Drüsengehalte der einzelnen Hautgebiete. Je geringer der Drüsenreichtum ist, um so größer ist die Disposition zur Lipombildung. Die symmetrische Anordnung erklärt sich durch die symmetrische Verteilung der Hautdrüsen. Die Produktion von Fett durch die Drüsen bildet den kausalen Zusammenhang. Störungen in der Innervation der Hautdrüsen mit konsequenter Verminderung der Sekretion führen zu Lipombildungen. Gegen diese Theorie wandten sich Borst, Köttnitz, Göbel, Askanacy und andere. Namentlich die tiefer gelegenen Lipome, die subfascialen und intramuskulären bleiben in ihrer Ätiologie von Grosch unaufgeklärt, da sie wegen ihres Sitzes von Hautdrüsen nicht abhängig sein können. Ebenso spricht die Rezidivfreiheit der Fettgeschwülste gegen Grosch. Köt t n it z spricht den Drüsen cine Fett sezernierende Eigenschaft ab. In sehr vielen Fällen werden trophoneurotische Störungen von Autoren als Ursache angesehen. Schon Grosch hat sie erwähnt. Gerade die sym- 
metrische Gruppierung der Lipome wird als ein Hinweis auf den nervösen Ursprung gedeutet. Köttnitz ist ein Anhänger dieser Theorie, viele andere haben weiterhin ihr ihre Anerkennung gezollt. A ls ber g beschreibt einen direkten Zusammenhang zwischen Lipom und Nervenästchen, den er bei der Untersuchung von mikroskopischen Schnitten entdeckt hat. Jedoch braucht ein Lipom, das einen Nerven umwächst, nicht von diesem ausgegangen zu sein (Borst). Zuletzt soll noch die Beziehung der Lymphdrüsen zur Lipombildung besprochen werden. Nach der Ansicht verschiedener Autoren sollen die Lymphdrüsen den Boden für die Lipombildung abgeben. Als Beleg führen sic die Substitution des Drüsengewebes durch Fettgewebe an, die sie in einigen Fällen nachweisen konnten (Göbel). A skanacy. konnte die Abstammung eincs Lipomes aus einer lipomatös umgewandelten anthrakotischen Lymphdrüse herleiten. Die Ergebnisse weiterer Untersuchungen von mesenterialen Lymphdrüsen führt er als Stütze seiner Theorie an. Hirschfeld, Aieolli nehmen ebenfalls einen Zusammenhang der Fettgeschwülste mit den Lymphdrüsen an. Borst erblickt für den Fall von Askanacy keine geschwulstmäßige Wucherung des Hilusfettes der Drüse, sondern nur eine exzessiv gesteigerte Zunahme.

Dies wären die hauptsächlichsten Erklärungsversuche, die man für die Entstehung der symmetrischen Lipome ersonnen hat. Es ist nicht leicht, sich bei der Erwägung der Ätiologie unserer zwei Fälle für eine bestimmte Theorie zu entscheiden. Jedenfalls verdient für beide Fälle vor allem eine embryonale Keimversprengung in Betracht gezogen zu werden, die durch uns unbekannte Einflüsse zum Wachstum angeregt zur Entstehung der Lipome geführt hat.

\section{Literaturverzeichnis.}

r. A ckermann, Beitr. z. Kenntnis u. Beurteilung d. mult. symm. Lip. Diss. Jena 1881 .

2. Aieoli, Über intime ätiol. u. pathog. Bezieh. zwisch. Lip., Adipos. usw. Ref. Münchn. med. Wochenschr. I906, S. 520.

3. A s kana cy, Zur Entstehung der mult. Lip. Virch. Arch. 1889 , Bd. 158, S. 407 . 
4. Blaschko, Eine seltene erbliche Lipombildung. Virch. Arch. 1891 , Bd. 124, S. 175 .

5. Horst; Die Lehre von den Geschwülsten. Bd. I, S. 134.

6. Braquehaye, Kong. Lip. des Rückens. Verb.-Zentr. f. allg. Path. u. path. Anat. I 895 , Bd. VI, S. 89.

7. Broer, Mult. symm. Lip. Ver-Ber. d. med. Wochenschr. 1go3, S. 6 t.

8. Rucquoy, Mult. symm. Lip. Ref. Monatsh. f. prakt. Derm. i\$93, S. 244 .

9. Is utlin, Fatty tum, in infancy and childhood. Ref. Zentrabl. $f$. Chir. $187 \delta$, S. 862.

Io. Chip a ult, Les lip. péricraniens. Ref. Zentralbl. f. Chir. I896, S. 489, II. D a rtig oll es, Mult. Lip. Ref. Monatsh. f. prakt. Derm. I893, S. 244. 12. De bove, Lipomat. dolorosa. Ref. Deutsche med. Wochenschr. I904, S. 1547 .

13. D e rt $1 \mathrm{ng}$ e r, Über tiefsitz. Lip. Beiträge z. klin. Chir. I903, Bd. 38 , S. 76 .

14. Doran, 4 Pfund schweres Lip. der Lumbalgegend. Ref. Schmidts Jahrb. 1899, Bd. 263 , S. 264.

15. Eckstein, Dem. eines Fall. $\checkmark$ mult. Lip. nach Trauma. Ver.-Ber. Münchn. med. Wochenschr. I906, S. I689.

I6. F.pstein, Mult. symm. I.ip. Ver.-Ber. Deutsche med. Wochenschr. 1907, S. 1517 .

17. Féré et Francillon, Note sur la coincidance des lip. symm. arec la paral. génér. progr. Ref. Zentralbl. f. Chir. J901, S, Io92.

18. F i eber, Ein Fall von mult. symm. Lip. Deutsche Zeitschr. f. Chir. I88 I, Bd. I4, S. 361 .

19. F is che r, Zur Lehre von den Lip. Arch. f. klin. Chir. Igo3, Bd. 69. S. 271 .

2o. Fle is cher, Fall von mult. symm. Lipomat. Sitzungsber. Berl. klin. Wochenschr. 1909, S. 1087.

2I. F́ ränke 1, Symm. Lipomat. Münchn. med. Wochenschr. I906, S. 527. 22. Göbe 1, Über mult. Lip. Zentralbl. f. allg. Path. u. path. Anat. 1895, Bd. 6, S. 4.

23. Grosch, Studien. über das Lip. Deutsche Zeitschr. f. Chir. I887, Bd. 26, S. 307 .

24. H irsch, Fall von hochgr. Lipomat. Sitz.-Ber. Berl. klin. Wochenschr. I9I I, S. 39.

25. Hirschfeld, Mann mit mult. Lip. Ver.-Ber. Münchn. med. Wochenschrift I903, S. 921 .

26. $\mathrm{H} \mathrm{ock}, 2$ Fälle von Lip. subconjunct. Prag. med. Wochenschr. I877, Nr. 10.

27. Hodges, Exceptional Ifatty tum. Zentralbl. f. Chir. 1885, S. 208.

28. Fï utchinson, Diffuse Lip. Ref. Berl. klin. Wochenschr. 19Io, S. 2402.

29. J a c o bi, Über kong. Lip. Ref. Zentralbl. f. Chir. 1884, S. 438.

30. Jaksch, Ein Beitrag z. d. Tum. d. Sehnenscheiden. Wiener med. Wochenschr. 1888, Nr. 36, S. I212. 
31. I s ra el. Disk. Tagebl. d. 59. Vers. deutsch. Naturf. u. Ärte I886, S. 150.

32. Klaußner, Ein wall ron Lip. Münchn. med. Wochenschr. 1895, Nr. 15, S. 349 .

33. Kleirkmecht, Über d. Lip. des Fußes. Beiträge z. klin. Chir. I898, Bd. 20, S. 335 .

34. Kleißel, Über mult. symm. Lip. Wiener med. Wochenschr. I912, Nr. 52, S. 3378 .

35. N linkowstein, Uber akute schmerzh. symm. Lipomat. Med. Klin. 1911, S. 1307 .

35. Kottnitz, C̈ber symm. Auftreten von Lip. Deutsche Zeitschr. f. Chir. 1894, Bcl. 38 , S. 75 .

37. Kölliker, Entwickl. eines Lip. nach Trauma. Zentralbl. f. Chir. 188 ;. S. 172 .

38. Kren, Symm. Lip. Dem. Ref. Wiener klin. Wochenschr. 1909, S. 944.

39. K üs t c r. Disk. Tagebl. d. 59. Vers. deutsch. Naturf. u. Ärzte I 886, S. I 50.

40. L a $\mathrm{g} \in \mathrm{r}$, Zur Kasuistik der mult. symm. Lip. Arch. f. klin. Chir. B.1. 46, S. 890 .

41. La uwers, Lip. du mésentère. Ref. Zentralbl. f. Chir. 189ı, S. 968.

42. L e normant et Verdun, Die symm. vorz. a. Halse vork. I.ipomat. Ref. Münchn. med. Wochenschr. I9ro, S. 1249.

43. L exer, Lehrbuch der aligem. Chir. Bd. 2, S. 256.

44. Löbker, Über einige Beobacht. $\therefore$ monströs. Lipombild. Zentralbl. f. Chir. 1884, S. 183.

45. v. Lutzau, Beiträge z. Kastistik d. mult. Lip. Diss. Dorpat. I 878 . Zentralbl. f. Chir. 1879, S. 205.

46. M a A u sland and Wood, lipomat. of the lower extr. with report of a case. Zentralbl. f. Chir. I9II, S. 1268.

47. Madelung, Uber den Fetthals. Arch. f. klin. Chir. 1888, Bd. 37, S. 106.

48. Marimón, Un caso de lipomat. trophon. consecut. á Trauma espinal. Ref. Zentralbl. f. Chir. I915, S, 4or.

49. Mors a line, Schilddrüsenbehandlung von Lip. Deutsche med. Wochenschrift 1909 , S. 988.

50. $\mathrm{M}$ üller, Ein Fall von diff. Lip. Arch. f. klin. Chir. 1889, Bd. 39, S. 652 .

51. Ne u welt, Morb. Based. u. Lipomat. Ref. Deutsche med. Wochenschr. $19 \mathrm{I} 3$, S. 331 .

52. Obtulowicz, Kong. Lip. enormer Größe. Zentralbl. f. Chir. I879, S. 892 .

53. Ochsner, Mult. symm. Lip. Zentralbl. f. Chir. 1897, S. 1319.

54. Pagenstecher, Lip. am inneren Fußrande. Ver.-Ber. Berl. klin. Wochenschr. I912, S. 2102.

55. Petrén, Ein Fall von mult. symm. heredit. Lip. Virch. Arch. 1897, Bd. 147, S. 560 .

56. P lettner, Beiträge z. Kenntn. d. tiefgel. Lip. Diss. Halle I889.

57. Rat hery et B in et, Symm. Iipomat. Sitzungsber. Berl. klin. Wochenschrift 1912, S. 2051. 
58. R ot h m a n n, Mult. symm. Lip. Ver.-Ber. Deutsche med. Wochenschr. 1904, S. 383 .

59. S a roum i a n, Contrib. à l'étude des lip. mult. symm. Ref. Lentralbl. f. Chir. 1908, S. 1527 .

6o. Savil1, Die Behandlung der Lip. mit Natriumäthyl. Ref. Münchn. med. Wochenschr. 1907, S. 1696.

61. Schemensky, Zur Kasuistik u. Ätiol. d. mult. symm. Lipomat. Münchn. med. Wochenschr. I912, S. I496.

62. Schuster, Ein Fall von mult. Lipomat. Kongr.-Ber. Berl. klin. Wochenschr. 1908, S. 1623 .

63. Sere nin, Über mult. symm. Lip. Ref. Zentralbl. f. Chir. I901, S. Ior8.

64. S in itzyn, Mult. Lip. Kongr.-Ber. Zentralbl. f. Chir. I896, S. 936.

65. S in $n$ h u e r, Fall v. mult. Lip. Ver.Ber. Deutsche med. Wochen. schrift 1904, S. 170.

66. Sp itzer, Mult. symm, Lip. Wiener med. Wochenschr. 1906, Nr. 15 , S. 714 .

67. v. Steyskel, Symm. Lipomat. u. Neurofibromat. Ver.-Ber. Deutsche ned. Wochenschr. 1907, S. 2165.

68. Derselbe, Symm. Lip. Ver.-Ber. Deutsche med. Wochenschr. I9o6, S. IIO2.

69. StrauB, Disk. Berl, klin. Wochenschr. Igog, S. 1088.

7o. Strümpell, Symm. Lipomat. Ver.-Ber. Münchn. med. Wochenschr. I903, S. 1185 .

71. Tém o in, Lip. périméningé simulant un spina bif. Ref. Zentralbl. f. Chir. 1893, S. 134 .

72. Th i $\mathrm{m} \mathrm{m}$, Adip. dolor. u. schmerzh. symm. Lip. Monatsh. f. pr. Derm. 1903 , Bd. 36, S. $28 \mathrm{r}$.

73. Tü rk, Symm. diffuse Lipomat. Ver.-Ber. Deutsche med. Wochenschr. 1904, S. 528.

74. Ders elbe, Diffuse symm. Lipomat. Ver.-Ber. Deutsche med. Wochenschr. I904, S. I952.

75. U m ber, Lipome. Sitzungsber. Berl. klin. Wochenschr. 1909, S. 375.

76. Virchow, Polysarcia lipomatodes. Ver.-Ber. Berl. klin. Wochenschr. I892, Nr. 27, S. 677.

77. Vossius, Uber einen Fall v. beiders. symm. Lip. am innern Augenwinkel. Kangr.-Ber. Med. Klinik 1913, S. 969.

78. Werner, Über kong. Lip. u. schwanzähnl. Bildungen b. Menschen. Virch. Arch. I908, Bd. I93, S. Iog.

79. Wolf sohn, Symm. Lipomat. Sitzungsber. Münchn. med. Wochenschrift 19I3, Nr. 45, S. 2543.

80. Wright, Stilbom foetus at full term .... Ref. Zentralbl. f. Chir. 1875, Bd. 2, S. 463 .

sı. $Z_{4}$ ckermann, Über ein knochenh. Lip. am Tub. ciner. Virch. Arch. I9II, Bd, 203, S. 157 . 